Z. vergl. Physiologie 74, 153-15̃5 (1971)

(C) by Springer-Verlag 1971

\title{
Collicular Responses to the Frequency Modulated Final Part of Echolocation Sounds in Rhinolophus ferrum equinum
}

\author{
G. Schuller, G. Neuweiler and H. U. Schnitzler \\ Zoophysiologisches Institut der Universität Tübingen
}

Received June 24, 1971

Summary. Collicular evoked potentials in Rhinolophus ferrum equinum show very prominent responses to the final frequency modulated part of a acoustic stimulus, simulating the natural echolocation sound.

The Greater Horseshoe Bat (Rhinolophus ferrum equinum) emits echolocation sounds consisting of a long part of constant frequency (cf) and a shorter final frequency modulated part (fm) sweeping down 14-16 $\mathrm{kHz}$. Flying bats lower the frequency in order to compensate for Dopplershift (Schnitzler, 1968). The echofrequency is therefore kept constant at about the frequency of the ef-part emitted by resting bats. The long ef-part is thought to be used for relative velocity measurements by Doppler-shifts, whereas it is still unknown if the short final fm-part renders any usefull information to the bat.

Nembutal anesthetized Horseshoe Bats ( $3 \mathrm{mg}$ Nembutal/100 g bodyweight) were stimulated by sounds of their own echotype simulated electronically by a trapezoid pulse generator (HP 8002 A), VCG-function generator (Wavetek 112) and an electronic switch. The ultrasonic speaker was placed $30^{\circ}$ above the plane of the bat's upper jaw and $30^{\circ}$ laterally to the body axis. Evoked potentials were recorded from the contralateral and ipsilateral colliculus inferior by Ag-AgCl-electrodes. The recorded signals were amplified by a differential amplifier (Tektronix 2A61). For every set of stimulus parameters 100 cyeles were summed up by a signal averager (Didac 800 Intertechnique) in order to improve signal to noise ratio.

The recorded collicular evoked responses show typical on-responses to the beginning and a very prominent off-response to the end of the simulated echolocation sound (Fig. $1 \mathrm{~b}$ ). In potentials evoked by pure ef-tones of $83.3 \mathrm{kHz}$ the off-response is hardly detectable or even missing (Fig. 1a). Thus the prominent off-response must be caused by the final fm-part of the echolocation sound.

11 Z. vergl. Physiologie, Bd. 74 


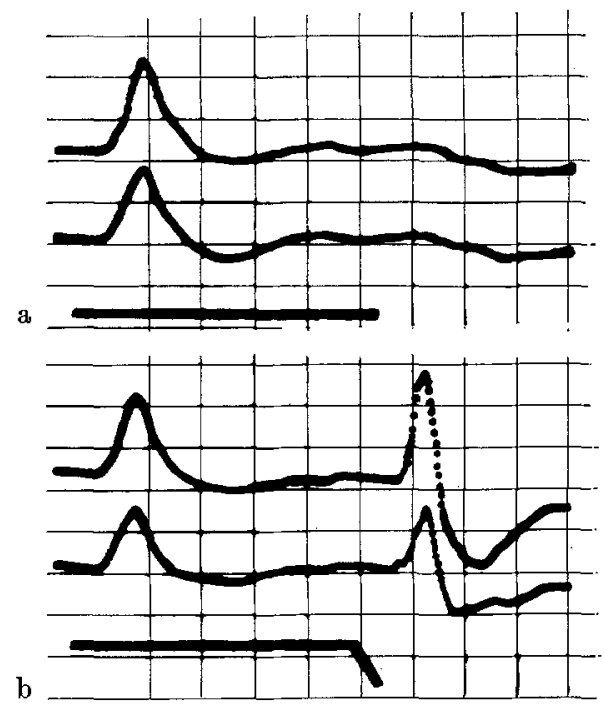

lig. $1 \mathrm{a}$ and $\mathrm{b}$. Responses of the colliculus inferior to echolocation sound without (a) and with (b) a final fm-part. Upper trace: contralateral; lower trace: ipsilateral. Stimulus: Cf-part $83.3 \mathrm{kHz}, 30 \mathrm{msec}$ duration; fm-part sweep from 83.3 to $67.3 \mathrm{kHz}$, 3 msec duration

This result contradicts the statement of Grinnell (1970) that in Chilonycteris, another bat using echolocation sounds with a long cf-part and a final fm-sweep, a sharp response to the final fm-part is absent. Grinnell explains the off-responses to echolocation sounds in Chilonycteris by two alternatives: 1 . the off-response is the true offresponse to the terminal constant frequency portion of the signal. As Fig. 1 shows this hypothesis is rejected by the results in Rhinolophus. 2. The off-response is elicited by the beginning of the fm-sweep. Our results in Rhinolophus indicate that the off-response is evoked not only by the beginning but by a substantial portion of the final $\mathrm{fm}$-sweep.

In any case the terminal fm-part of the echolocation sound in Rhinolophus causing such clear cut responses in the acoustical center cannot be considered as a mere by-product of sound generation. Vespertilionid bats use for echolocation only fm pulses ressembling the fm-part of Rhinolophid sounds in duration and intensity. We suppose that Horseshoe Bats get substantial echoinformation out of the final fm-part of the sound.

A detailed analysis of collicular responses to frequency modulated parameters is currently undertaken. 


\title{
References
}

Grinnell, A. D.: Comparative auditory neurophysiology of neotropical bats employing different echolocation signals. Z. vergl. Physiol. 68, 117-153 (1970).

Schnitzler, H.-U.: Die Ultraschall-Ortungslaute der Hufeisen-Fledermäuse (Chiroptera-Rhinolophidae) in verschiedenen Orientierungssituationen. Z. vergl. Physiol. 57, 376-408 (1968).

\author{
G. Schuller \\ Dr. G. Neuweiler \\ Dr. H. U. Schnitzler \\ Zoophysiologisches Institut \\ Arbeitsgruppe Elektrophysiologie \\ BRD-74 Tübingen, Köllestraße 23 \\ Germany
}

